\title{
MAGNETIZATION TRANSFER CONTRAST IMAGING OF HEPATIC NEOPLASMS
}

\author{
Michael D. Hollett, Alex M. Aisen, Hong N. Yeung, \\ ISAaC R. Francis, and Robert L. BreE \\ Department of Radiology, University of Michigan Medical Center, Ann Arbor, MI 48109-0030, USA
}

\begin{abstract}
A method of performing magnetization transfer contrast (MTC) using a pulse sequence based on a series of onresonance binomial pulses preceding a conventional spin-echo sequence has been recently described. We investigated this technique in the evaluation of circumscribed hepatic neoplasms on a $0.5 \mathrm{~T}$ imager. Conventional spin-echo imaging was performed in 18 patients with hepatic neoplasms, 15 with primary or metastatic hepatic neoplasms, and 3 with cavernous hemangiomas. The $T_{1}$-weighted sequence was repeated following MTC saturation in 7 patients and the proton density sequence was repeated following MTC saturation in 13 patients (both were used in two patients). The mean lesion-to-liver contrast for neoplasms worsened with $T_{1}-\mathrm{MTC}(0.87$ vs. 1.04), but improved slightly with proton density-MTC (1.10 vs. 1.22) when compared to the corresponding non-MTC spinecho images. However, these differences were not statistically significant ( $p=.19$ and $p=.16$, respectively). The limited data on cavernous hemangiomas does not suggest these have consistently different MTC imaging characteristics from other hepatic neoplasms. In conclusion, MTC imaging using a binomial saturation pulse did not offer significant improvement in contrast between hepatic neoplasms and normal liver. Refinements of the binomial pulse sequence will be necessary if a consistent improvement in lesion sensitivity is to be achieved. Evaluation of its role in increasing diagnostic specificity will require further investigation.
\end{abstract}

Keywords: MRI; Magnetization transfer contrast; Liver metastases.

\section{INTRODUCTION}

For purposes of MR imaging, biological tissue contains two types of hydrogen atoms: those in the "liquid" state with relatively long relaxation times, which directly contribute to signal on MR images, and those in the "solid" state which have very short $T_{2}$ times and do not provide a direct contribution. Water and non-membrane fat fall in the former category, while macromolecular hydrogen falls in the latter. Though macromolecular hydrogen does not contribute directly to MR signal, its presence can be manifest by its influence on the magnetic properties of surrounding water. Magnetization transfer contrast (MTC) imaging is a recently described modification of conventional MR imaging in which the magnetization of short- $T_{2}$ macromolecular hydrogen atoms is selectively saturated prior to a standard pulse sequence. ${ }^{1}$ This results in an alteration in the MR properties of water protons, specifically, a reduction in the steady-state magnetization and a decrease in the apparent $T_{1}{ }^{1,2}$ MTC has been proposed as a method of enhancing image contrast and tissue characterization. ${ }^{3-6}$

The saturation of the macromolecular hydrogen atoms has most commonly been implemented by application of a continuous off-resonance radio frequency (RF) pulse $(\Delta \omega=1.5-10 \mathrm{kHz})$ with a separate RF channel. ${ }^{1,3-6}$ There are several disadvantages of this method, including the requirement of additional hardware and, at higher field strengths, RF energy deposition considerations. An alternative method, originally proposed by $\mathrm{Hu}$ et al. and recently described by Yeung and Aisen, employs a train of on-resonance $(\Delta \omega=0)$ binomial pulses to saturate the macromolecular hydrogen atoms. ${ }^{7-9}$

While both methods achieve selective magnetic saturation of macromolecules based on their rapid $T_{2}$ relaxation, this is accomplished through entirely different means with similar but not identical results. With the continuous irradiation technique, one takes advantage of the broadening of the resonance spectrum of the macromolecules by delivering the RF power away from 
the water resonance peak. This results in a better selectivity at the expense of efficiency. The pulse technique prevents the direct saturation of the water protons by applying on-resonance RF pulses with a binomial shape and hence zero net tip angle., ${ }^{9,10}$ Protons with long relaxation times compared to the binomial pulse width are relatively unaffected by the pulse, whereas macromolecular protons with short relaxation times do not "see" both halves of the pulse symmetrically, and are thus gradually saturated by a series of such pulses. The binomial pulse technique has the advantage of ease of implementation and higher RF efficiency (measured by the degree of saturation per watt of RF power), but at the price of some degree of nonspecific signal attenuation, which depends on the transverse relaxation time of the specific tissue.

The purpose of this study was to investigate the utility of pulsed MTC in the evaluation of hepatic neoplasms. The goals were: 1) to evaluate the signal characteristics of hepatic neoplasms with respect to normal liver using the MTC sequence (with a conventional spin-echo sequence as a control); and 2) to determine if MTC can improve lesion detectability.

\section{METHODS}

Eighteen patients with at least one hepatic mass $\geq 2 \mathrm{~cm}$ were studied. Fifteen patients had pathologically proven hepatic neoplasms: seven metastatic adenocarcinoma of the colon, two metastatic carcinoid, two hepatomas, one hepatic adenoma, one metastatic prostate carcinoma, one metastatic transitional cell carcinoma, and one metastatic adenocarcinoma of the pancreas. For the purposes of this study, the one hepatic adenoma is included in the group of primary and metastatic malignant neoplasms. Three patients had cavernous hemangiomas as determined by known CT and MR criteria. ${ }^{11}$ Informed consent was obtained from all subjects.

Imaging was performed on a 0.5-T MR unit (Vista HP, Picker International, Highland Heights, Ohio, USA). The manufacturer's supplied body coil was used for both RF transmission and as a receiver coil in all studies with the exception of one case in which a surface coil was used as the receiver. Initially, multisection spin-echo imaging of the liver using $T_{1}$ (TR = $500-633 \mathrm{~ms}$, TE $=12-20 \mathrm{~ms})$, proton-density (2000$2500 \mathrm{~ms} / 20-30 \mathrm{~ms})$ and $T_{2}(2000-2500 \mathrm{~ms} / 70-80 \mathrm{~ms})$ weighted spin-echo sequences was performed. Subsequently, either one or four contiguous tomographic images were obtained at the level of the lesion using a binomial MTC spin-echo sequence. The MTC pulse sequence employed a $400 \mathrm{~ms}$ saturation pulse (consisting of a train of sixteen $5 \mathrm{~ms}$ on-resonance $1 \overline{1}$ - binomial pulses with a $20 \mathrm{~ms}$ interval between pulses) followed by a conventional spin-echo ( $T_{1}$ or proton density) sequence. The nature and efficacy of the binomial MTC pulse sequence used has been reported previously. The field of view was $34-42 \mathrm{~cm}$, matrix size $256 \times 256$ or $256 \times 192$, and section thickness was 7.5 or $10 \mathrm{~mm}$.

Details of the timing parameters of the MTC sequences and controls for each group are as follows. The first seven patients (all with malignant hepatic neoplasms) were studied with a $T_{1}$-MTC sequence. In six of these, a $T_{1}$-MTC $(600 / 20)$ image was compared with the corresponding $600 / 20$ spin-echo image from a multisection examination; a $T_{1}$-MTC $(533 / 20)$ image was compared to a $500 / 12$ spin-echo image in one case. Thirteen patients (including two patients from the first group) were studied with a proton density-MTC sequence. In 11 cases, a proton density-MTC (2000/20) image was compared with the corresponding $2000 / 20$ spin-echo image from a multisection examination; the proton density-MTC $(2000 / 20)$ image was compared with an image from a multisection examination with slightly different timing parameters (2000/30 and 2250/ $20)$ in two cases. These small variations were not considered to be important to the analysis.

Region of interest measurements were performed using the manufacturer's software to compare the signal intensity ratio of the hepatic mass to a normal region of the liver at the same level. Intensities were normalized for receiver gain settings, image display normalization factor, field of view, slice thickness, and the use of anti-aliasing. Noise signal (air) was also recorded and normalized for matrix size and the number of signal accumulations. The ratios were calculated for both MTC sequences employed and the corresponding conventional SE sequences. The pairs of signal intensity ratios from each group were compared using Student's $t$-test (paired two-tail).

To determine the degree of MTC-specific and nonspecific signal attenuation, tissue to control (i.e., MTC/ non-MTC) intensity ratios were calculated for normal liver, lesion (malignant neoplasms and hemangiomas), muscle, and fat. Since fat is essentially unaffected by MTC, changes in its signal largely reflect nonspecific signal loss. Signal-to-background noise (air) ratios for liver on non-MTC and MTC sequences are also presented to demonstrate changes in image quality with MTC. For these measurements, noise was taken as the mean signal intensity of an ROI drawn in the background, as far from phase-encoding artifact as practical, and normalized to a standard reference to correct for acquisition parameters. As such, it differs from the standard deviation measurement often used, but is adequate for this purpose.

Qualitative analysis was also performed, comparing the $T_{1}$ - or proton density-MTC image(s) to the con- 
ventional $T_{1}$-weighted, proton density-weighted, and $T_{2}$-weighted spin-echo images. The images were appropriately windowed by an experienced technologist. For each case, the distinctness of each lesion on the MTC image was graded by consensus of two of the authors as 1) inferior to the similarly weighted non-MTC control sequence, 2) equivalent to the control sequence, 3) superior to the control sequence, or 4) superior to the control and all other spin-echo sequences. Thus, only in the latter case was the MTC sequence judged to provide the best depiction of the lesion of all pulse sequences we used.

\section{RESULTS}

Two cases demonstrating the effects of MTC irradiation are shown in Figs. 1 and 2. Figure 1 demonstrates an improvement in lesion-to-liver contrast for a hemangioma on the proton density-MTC image compared with the non-MTC proton density image, but image quality (signal-to-noise) is diminished. The best

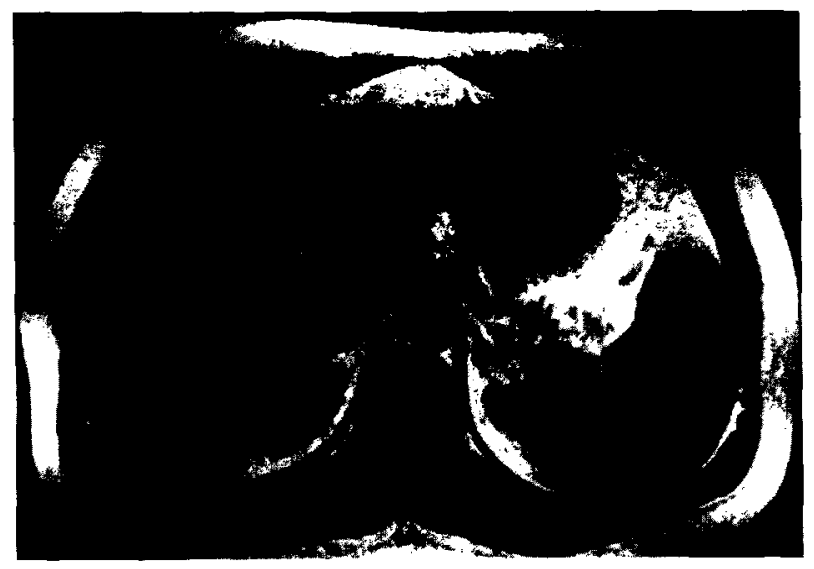

(A)

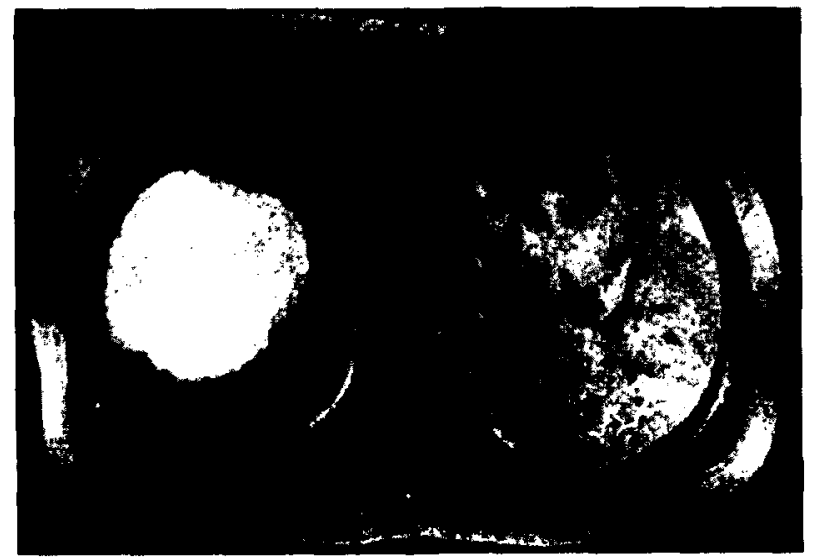

(C) contrast is on the conventional $T_{2}$-weighted image. Figure 2 is a patient with metastatic adenocarcinoma from the prostate. This examination shows the lack of contrast improvement for malignant neoplasms with proton density-MTC imaging. Although there is a noticeable reduction in signal from normal liver and muscle relative to fat, lesion-to-liver contrast is nearly the same when compared to the non-MTC proton density image.

The signal intensity ratios for each MTC sequence and the conventional spin-echo sequences are presented in Table 1. The mean lesion-to-liver contrast for malignant hepatic neoplasms worsened with $T_{1}$-MTC $(0.87$ vs. 1.04$)$, but improved slightly with proton density-MTC ( 1.10 vs. 1.22$)$ when compared to the corresponding non-MTC spin-echo images. However, the differences were not statistically significant $(p=.19$ and .16, respectively). Although the limited data for hemangiomas suggests an improvement in contrast (1.21 vs. 1.64), the data are limited and the results were not consistent. The individual changes in signal inten-

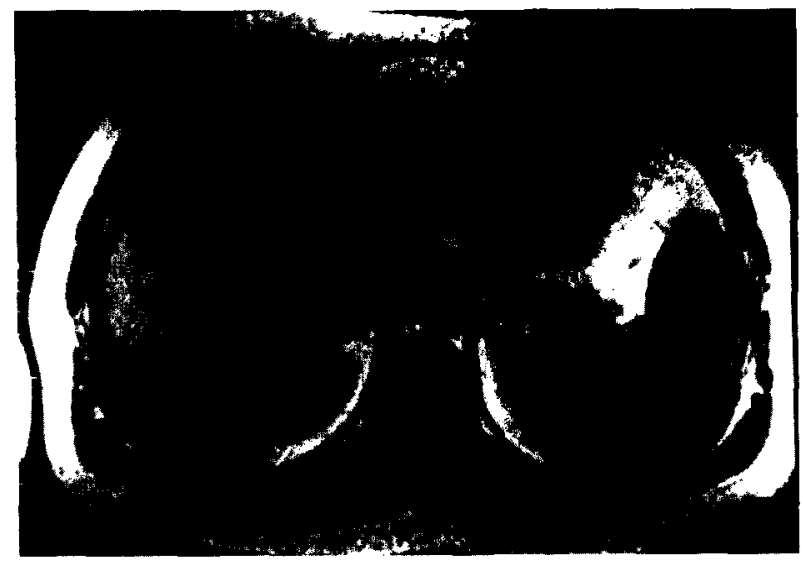

(B)

Fig. 1. A 38-yr-old male with a large hemangioma in the right lobe of the liver. (A) Proton density-weighted and (B) proton density-MTC images. The contrast between the hemangioma and normal liver is slightly improved. However, the overall image quality is reduced as a result of the lower signalto-noise. (C) $T_{2}$-weighted image. Lesion contrast is greatest on the conventional $T_{2}$-weighted image. 


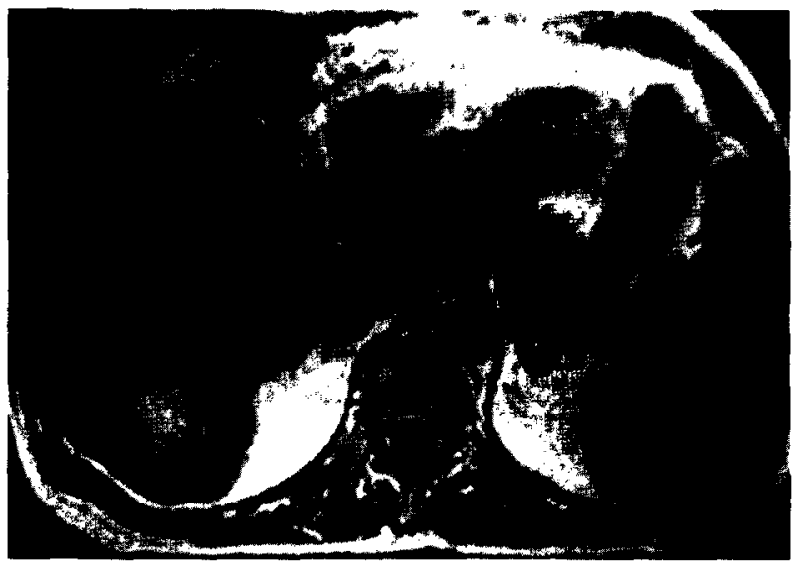

(A)

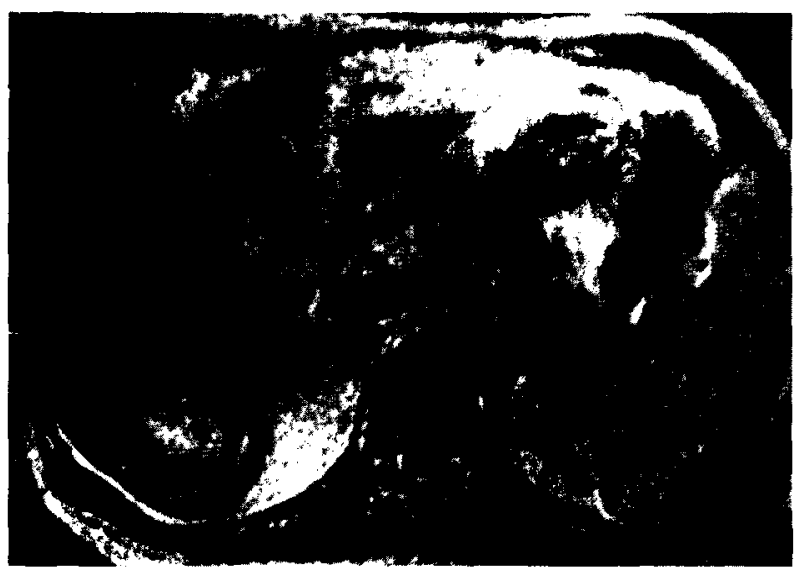

(C)

sity ratio with MTC compared to the corresponding control sequence are illustrated by group in Figs. 3A and 3B. Note that the hemangioma-to-liver contrast using the proton density sequence demonstrate an improvement in contrast in one case, but in the two other cases the improvement in lesion-to-liver contrast was negligible.

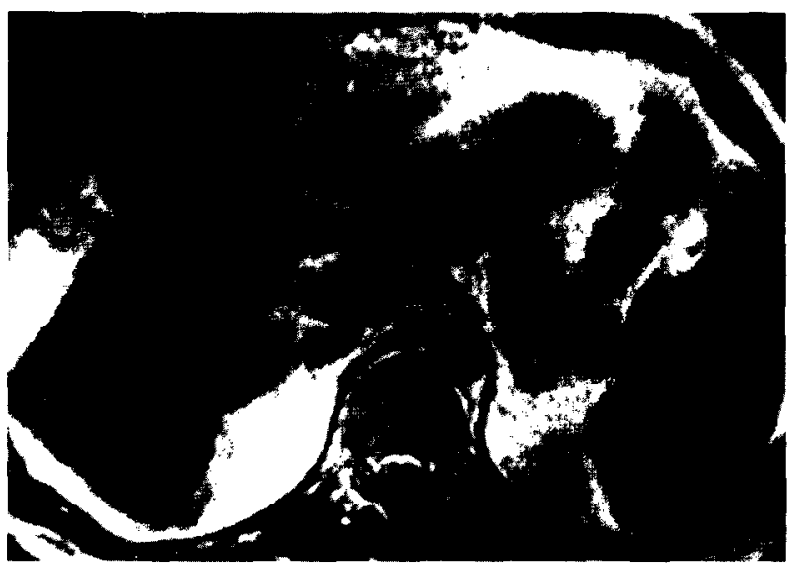

(B)

Fig. 2. A 69-yr-old male with metastatic adenocarcinoma of the prostate in the posterior segment of the right lobe of the liver (arrow). (A) Proton density-weighted and (B) proton density-MTC images. With MTC, the expected reduction in the signal from normal liver and muscle relative to fat is noticeable. However, lesion-to-liver contrast does not change significantly. (C) $T_{2}$-weighted image. Lesion contrast is greatest on the conventional $T_{2}$-weighted image.

Table 1. Mean lesion-to-liver contrast shown for each patient group

\begin{tabular}{lccccc}
\hline & \multicolumn{5}{c}{ Intensity ratios: Lesion/liver (mean \pm SD) } \\
\cline { 2 - 6 } & $T_{1}$ & $T_{1}$-MTC & PD & PD-MTC & $T_{2}$ \\
\hline Group 1: Malignant neoplasmas $(n=7)$ & $\mathbf{0 . 8 7} \pm \mathbf{0 . 2 6}$ & $\mathbf{1 . 0 4} \pm \mathbf{0 . 2 3}$ & $1.12 \pm 0.13$ & & $1.58 \pm 0.54$ \\
Group 2: Malignant neoplasms $(n=10) *$ & $0.92 \pm 0.22$ & & $\mathbf{1 . 1 0} \pm \mathbf{0 . 1 5}$ & $\mathbf{1 . 2 2} \pm \mathbf{0 . 2 7}$ & $1.54 \pm 0.36$ \\
Hemangiomas $(n=3)$ & $\mathbf{0 . 7 0 \pm 0 . 0 4}$ & & $\mathbf{1 . 2 1} \pm \mathbf{0 . 0 6}$ & $\mathbf{1 . 6 4} \pm \mathbf{0 . 7 0}$ & $2.23 \pm 0.28$ \\
\hline
\end{tabular}

*Includes one hepatic adenoma.

The closer the ratio is to unity, the less conspicuous the lesion. Data for which MTC and conventional spin-echo sequences are compared emphasized in bold.

Signal intensity ratios for specific tissues with MTC compared to the corresponding control (spin-echo) sequences are shown in Figs. 4A and 4B. Note that there is a decrease in the signal for fat of $33-34 \%$. This change is nonspecific, because fat protons do not spin-couple to macromolecules, and thus do not undergo substantial MTC effect. However, fat protons have moderate 


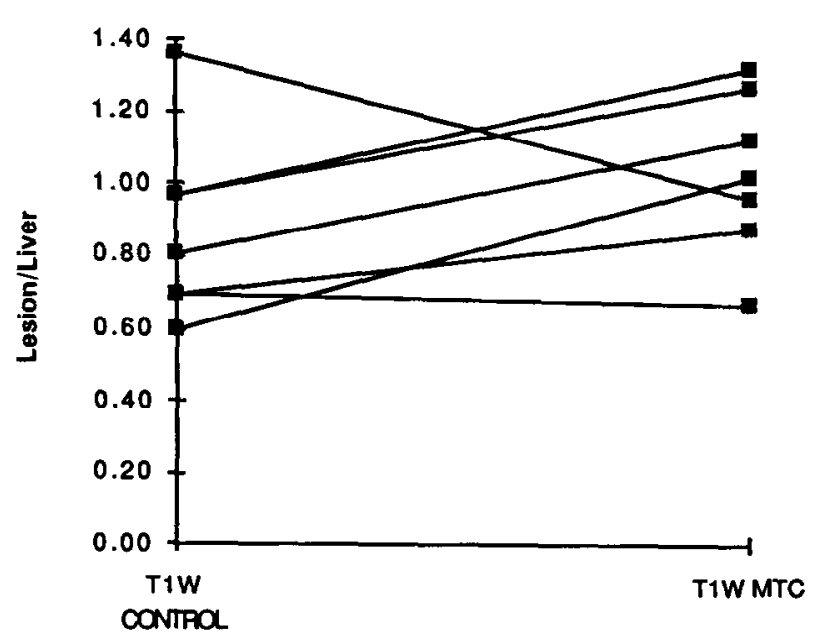

(A)

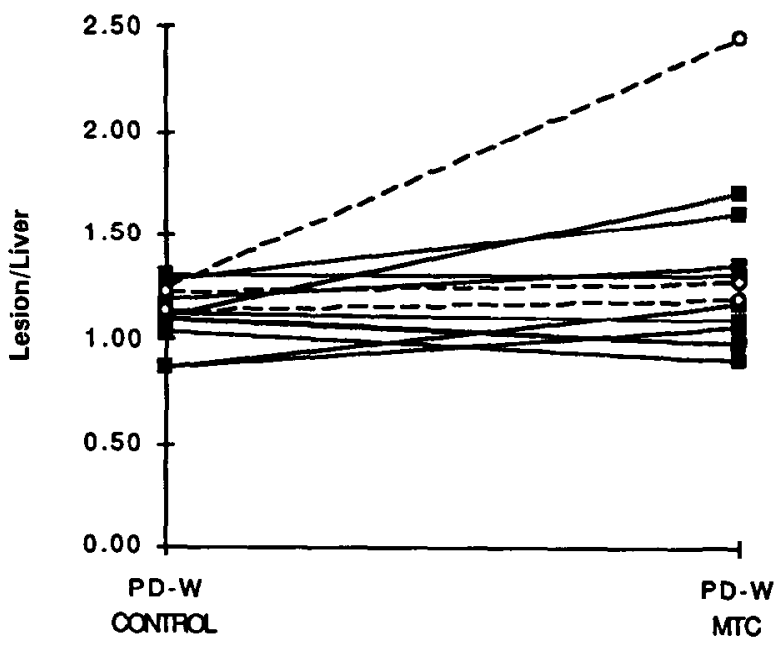

(B)

Fig. 3. Liver to normal liver signal intensity ratios. (A) $T_{1}$-MTC. There was no improvement in the image contrast for malignant hepatic neoplasms with MTC compared to the $T_{1}$-weighted control. (B) Proton density-MTC. Solid lines indicate patients with malignant hepatic neoplasms (includes one hepatic adenoma). Dashed lines indicate patients with hemangiomas. There was no consistent improvement in lesion contrast with MTC for malignant neoplasms nor hemangiomas. Note signal intensity ratio increased significantly for hemangioma in one case (same patient as Fig. 1). (Note: Nearly identical data in two cases $(1.10,0.98)$ are superimposed).

to short transverse relaxation times, resulting in nonspecific signal loss using this sequence. As expected, the signal loss for muscle is the greatest of the tested tissues. Muscle has a high protein content, and has been found to undergo dramatic signal suppression with MTC irradiation.' The signal change in muscle results from the combined nonspecific signal loss that affects fat and specific MTC effect. The signal changes for
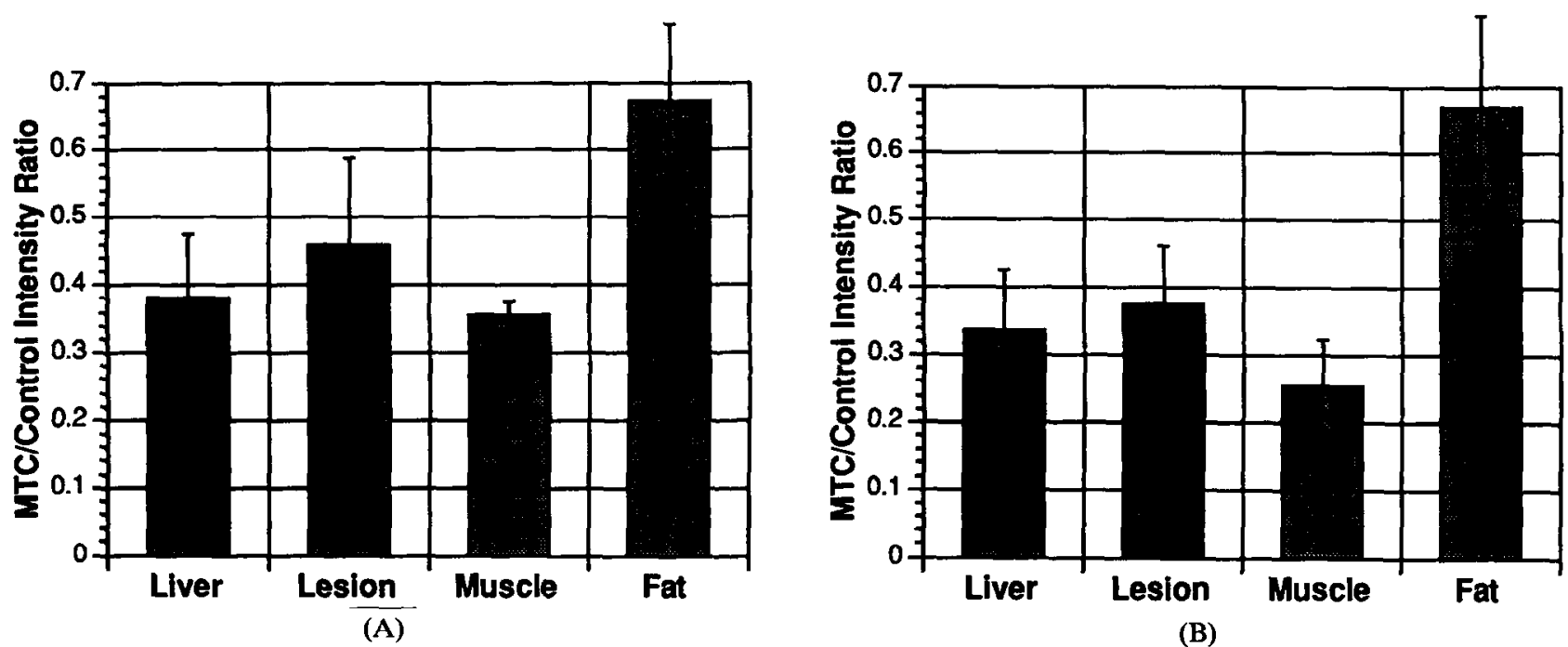

(B)

Fig. 4. Tissue-specific MTC effects. Normalized signal intensity for each tissue with MTC compared to the corresponding control sequence. (A) $T_{1}$-MTC $(n=7)$ and (B) proton density-MTC $(n=13)$ ratios. Fat undergoes relatively little MTC effect (see text), hence the decrease in the fat ratio from unity reflects nonspecific signal loss. Muscle is affected to the greatest degree. Note that the changes are greater for the proton density-MTC group than for the $T_{1}$-MTC group due to the counterbalancing effects of $T_{1}$-MTC. 
liver and lesion were similar, and intermediate between muscle and fat.

Also note that the signal losses on the $T_{1}$-weighted images were less than on the proton density sequences. This is expected as MTC irradiation will tend to shorten $T_{1}$ times; on the $T_{1}$-weighted sequences this will increase signal and counteract the MTC-induced signal loss from reduction of the magnetization.

The subjective evaluation of image contrast is limited by the confounding effects of windowing, but is provided for completeness. Image contrast improved on the $T_{1}$-MTC sequence following saturation in only one case (metastatic prostate carcinoma), but was inferior to the $T_{2}$-weighted sequence. In the other six cases, image contrast with magnetization transfer was inferior to the control $T_{1}$-weighted spin-echo sequence.

In the proton density-MTC group, image contrast was superior to both the control spin-echo sequence and the standard $T_{2}$ sequence in two cases, one with metastatic adenocarcinoma of the colon and the other with a hepatic adenoma. Image contrast improved on MTC images in three additional cases (one hepatoma and two hemangiomas) compared with the proton density control image, but in each of these cases the conventional $T_{1}$ and/or $T_{2}$ sequence was superior. In the remaining eight cases, subjective image contrast was either unchanged (five cases) or inferior (three cases) to the control.

In all cases, the signal-to-noise ratio decreased as a result of the specific and nonspecific signal attenuation inherent in this MTC sequence. For the $T_{1}$-MTC images, the signal-to-noise was $5.0 \pm 1.0$ vs. $11.0 \pm 3.5$ for the $T_{1}$-weighted control spin-echo images. For proton density-MTC, the signal-to-noise was $5.4 \pm \mathbf{2 . 0}$ vs. $16.0 \pm 7.3$ for the proton density-weighted spin-echo images. This was readily apparent as a decrease in image quality when the examinations were evaluated subjectively. Thus, even for those cases with an improvement in lesion contrast, lesion detectability was decreased.

\section{DISCUSSION}

There has recently been significant interest in magnetization transfer effects in MR images for several reasons. ${ }^{1,3-6}$ First, work with the technique promises to yield significant insight to the mechanisms responsible for transverse and longitudinal relaxation, and thus help elucidate the underlying biophysical mechanisms that produce tissue contrast on MR images. Second, there has been some initial hope that MTC pulse sequences may improve image contrast in certain diagnostic situations. ${ }^{1,3-5,12}$ Third, MTC sequences may contribute to diagnostic specificity in some situations, as different tissue types may undergo characteristic signal alterations. ${ }^{1,3,4,6,11}$ Fourth, significant MTC effects may be present "accidentally" in conventional imaging sequences, particularly those which employ many RF pulses. ${ }^{13,14}$ Fifth, MTC effects may be useful as a means of deliberately suppressing tissue signal, for example, to increase blood-static tissue signal intensity ratios in vascular sequences. The aim for this study was to address the second point and provide some information towards the third.

Initial work by Wolff et al. demonstrated that imaging the knee with a gradient-echo-MTC sequence increased the cartilage-synovial fluid and muscle-fat contrast. ${ }^{4}$ This effect was subsequently shown in the heart, with increased contrast between myocardium and ventricular blood as a consequence of MTC-induced attenuation of myocardial signal with little change in the bright blood signal. ${ }^{5}$ An initial application of MTC to the liver on a 1.5-T scanner found that MTC did not improve lesion-to-liver contrast in most cases; however, there was a contrast increase for cysts and hemangiomas. ${ }^{6}$ Conversely, an investigation using a 0.1-T instrument applied to 17 patients with malignant liver lesions did show contrast improvement. ${ }^{15}$ This discrepancy suggests that MTC may be of more use at lower field strengths than higher ones. Our own results are more consistent with the 1.5-T work.

This study differs from most earlier work in that we used a series of on-resonance binomial RF pulses to produce the saturation of macromolecular hydrogen nuclei necessary to produce MTC. ${ }^{7-10}$ This sequence is easier to implement than the common alternative, a longer, continuous, off-resonance RF irradiation. The binomial sequence also deposits less RF energy in the patient, an important consideration at higher field strengths. On the other hand, it produces some degree of nonspecific signal loss which degrades image quality. Further, while most of the signal changes with this sequence are believed to be due to magnetization transfer, it is possible that some of the effect we (and others) are attributing to MTC is really a result of direct saturation of water protons, a non-MTC phenomenon.

In the first seven patients in this study, MTC was performed using $T_{1}$-weighted images. This decision was motivated by the fact that the MTC predominantly affects longitudinal magnetization and thus alters $T_{1}$. The $T_{1}$ shortening observed with MTC can be expected to increase relative signal on $T_{1}$-weighted images. However, MTC also lowers the "steady-state" magnetization $\left(M^{*}\right)$, which has the opposite effect on overall signal. To better demonstrate the latter effect, unmitigated by the former, we performed MTC with a proton density sequence in the remaining patients. Tissues susceptible to the MTC phenomenon will show 
a relatively larger decrease in signal on the proton density-MTC images. This is illustrated by the increased liver to muscle contrast resulting from the comparatively greater decrease in signal from the protein-rich muscle tissue.

Lesion-to-liver contrast for the hepatic neoplasms was not consistently improved with either MTC sequence employed. The lack of contrast improvement suggests that the pulsed saturation method does not affect normal liver in a manner significantly different from the hepatic neoplasms studied. These results were in accord with the subjective image analysis, although the qualitative findings are somewhat confounded by decreased signal-to-noise for the MTC images and variations in windowing.

The hemangiomas are a special case because they have MR characteristics similar to fluid. One would expect, as Outwater et al. observed, that their signal would be relatively unaffected by MTC, while that of normal hepatic parenchyma would be reduced. ${ }^{6}$ In only one of the three hemangiomas imaged in this series was the expected change in relative signal intensity observed. This suggests MTC may not be reliable in differentiating between hemangiomas and solid hepatic neoplasms, but firm conclusions should not be drawn from such limited data.

Signal-to-noise and, as a consequence, overall image quality was diminished with MTC imaging. The decreased image quality is a consequence of both the specific effects of MTC and the undesirable but unavoidable nonspecific signal loss that occurs with the binomial pulse sequence. The combined effects can be estimated from the loss in signal-to-noise for liver, while the nonspecific losses are documented by the changes in the signal-to-noise of fat (since fat has a somewhat shorter $T_{2}$ time than many other tissues, the nonspecific signal loss with pulsed MTC may be higher than with other tissues). This loss in image quality is serious, and is likely to limit the usefulness of MTC techniques, especially at lower field strengths. Higher order binomial pulses (e.g., $1 \overline{2} 1$ ) also produce such nonselective signal loss, to roughly the same degree for equivalent duty cycle. We note, however, that since this work was completed, enhancements to the binomial sequence, such as increasing power and shortening pulse width, have been demonstrated; these may reduce nonspecific signal loss. ${ }^{10}$

Higher field strength examinations with substantially better signal-to-noise may permit more accurate quantitative evaluation and tissue differentiation. Other important areas of tissue characterization also warrant further evaluation, for instance, the evaluation of fibrosis.

\section{CONCLUSION}

In this series of 18 patients with hepatic neoplasms we found that $T_{1}$-MTC resulted in slight worsening in lesion-to-liver contrast when compared to the corresponding control spin-echo sequence; slight improvement occurred with proton density-MTC compared to controls. However, the findings were neither statistically significant nor consistent from case to case. In addition, the reduced signal-to-noise related to the binomial saturation pulse significantly reduced image quality. This most often negated any improvement in contrast when the images were evaluated for lesion conspiquity. Improved binomial pulse design is likely to lead to an improvement in MTC image quality. Combined with imaging at higher field strengths lesion detection may eventually be improved in some cases. In three cases, we found no consistent evidence that the addition of MTC can reliably diagnose hepatic cavernous hemangiomas, though further work is justified.

\section{REFERENCES}

1. Wolff, S.D.; Balaban, R.S. Magnetization transfer contrast (MTC) and tissue water proton relaxation in vivo. Magn. Reson. Med. 10:135-144; 1989.

2. Grad, J.; Mendelson, D.; Hyder, F.; Bryant, R.G. Direct measurements of longitudinal relaxation and magnetization transfer in heterogeneous systems. J. Magn. Reson. 86:416-419; 1990.

3. Wolff, S.D.; Eng, J.; Balaban, R.S. Magnetization transfer contrast: Method for improving contrast in gradientrecalled-echo images. Radiology 179:133-137; 1991.

4. Wolff, S.D.; Chesnick, S.; Frank, J.A.; Lim, K.O.; Balaban, R.S. Magnetization transfer contrast imaging of the knee. Radiology 179:623-628; 1991.

5. Balaban, R.S.; Chesnick, S.; Hedges, K.; Samaha, F.; Heineman, F.W. Magnetization transfer contrast imaging of the heart. Radiology 180:671-675; 1991.

6. Outwater, E.; Schnall, M.D.; Braitman, L.E.; Dinsmore, B.J.; Kressel, H.Y. Magnetization transfer of hepatic lesions: Evaluation of a novel contrast technique in the abdomen. Radiology 182:535-540; 1992.

7. Hu, B.S.; Nishimura, D.; Macovski, A. Pulsed magnetization transfer contrast (astr). In: Book of abstracts: Ninth Annual Meeting of the Society of Magnetic Resonance in Medicine. Berkeley, CA:SMRM; 1990: 352.

8. Hu, B.S.; Conolly, S.M.; Wright, G.A.; Nishimura, D.G.; Macovski, A. Pulsed saturation transfer contrast. Magn. Reson. Med. 26:231-240; 1992.

9. Yeung, H.N.; Aisen, A.M. Magnetization transfer contrast with periodic pulsed saturation. Radiology 183:209$214 ; 1992$.

10. Yeung, H.N. Transient response of a heterogeneous spin system to binomial pulse saturation. J. Magn. Reson. A102:8-15; 1993. 
11. Berland, L.; Lee, J.K.T.; Stanley, R.J. Liver and biliary tract. In: J.K.T. Lee, S.S. Sagel, R.J. Stanley (Eds). Computed Body Tomography With MRI Correlation, 2nd ed. New York: Raven Press; 1989: pp. 610-615.

12. Dixon, W.T. Use of magnetization transfer contrast in gradient-recalled echo images. Radiology 179:15-16; 1991.

13. Dixon, W.T.; Engels, H.; Castillo, M.; Sardahsti, M. Incidental magnetization transfer contrast in standard multislice imaging. Magn. Reson. Imaging 8:417-422; 1990.

14. Mulkern, R.V.; Melki, P.S.; Lilly, H.S. Magnetization transfer contrast with multisection RARE sequences (abstract). J. Magn. Reson. Imaging 2:55; 1992.

15. Kahn, C.E., Jr.; Perera, S.D.; Sepponen, R.E.; Tanttu, J.I.; Tierala, E.K.; Lipton, M.J. Magnetization transfer imaging of the abdomen at $0.1 \mathrm{~T}$ : Detection of hepatic neoplasms. Magn. Reson. Imaging 11:67-71; 1993. 